\title{
Los operadores escalares en el español hablado de Santiago de Chile: análisis pragmático y sociolingüístico de sobre todo, al menos y por lo menos ${ }^{1}$
}

\section{Scalar operators in the spoken spanish of Santiago, Chile: pragmatic and sociolinguistic analysis of sobre todo, al menos and por lo menos}

\section{ABELARDO SAN MARTÍN NÚNEEZ CRISTIAN ROJAS INOSTROZA}

\author{
Universidad de Chile, Facultad de Filosofía y Humanidades, \\ Departamento de Lingüística. Santiago, Chile. \\ Correo electrónico: asmartin@uchile.cl \\ Universidad de Chile, Facultad de Filosofía y Humanidades, \\ Departamento de Lingüística. Santiago, Chile. \\ Correo electrónico: rojascristian@ug.uchile.cl
}

\begin{abstract}
En este estudio se describe el comportamiento pragmático y sociolingüístico de los operadores escalares sobre todo, al menos y por lo menos en un corpus de 120 entrevistas sociolingüísticas del español de Santiago. El análisis pragmático profundiza en el carácter polifuncional de estas unidades con base en sus valores en los distintos planos de la actividad discursiva (argumentativo, enunciativo, informativo y modal), además de explorar el papel "intensificador" de sobre todo y la codificación de "vaguedad enunciativa" de al menos y por lo menos. El análisis sociolingüístico arrojó una distribución social relativamente homogénea de sobre todo, mientras que el empleo de por lo menos es prominente entre los hablantes del primer grupo etario y los del grupo socioeconómico medio alto y al menos, por su parte, es empleado mayoritariamente por hablantes del segundo grupo etario y del grupo socioeconómico bajo. Con todo, solo la variación de por lo menos resultó significativa estadísticamente.
\end{abstract}

Palabras clave: operadores escalares, planos textuales, polifuncionalidad, español de Chile.

\footnotetext{
${ }^{1}$ Este estudio forma parte del proyecto FONDECYT Regular N¹161422, "Los marcadores del discurso en el español hablado en Santiago de Chile: análisis pragmático y sociolingüístico”.
} 
This study describes the pragmatic and sociolinguistic behavior of scalar operators sobre todo, al menos and por lo menos in a corpus of 120 sociolinguistic interviews of the Santiago, Chile spanish. The pragmatic analysis deepens in the polyfunctional character of these units based on their values in the different levels of the discursive activity (argumentative, enunciative, informative and modal), besides exploring the "intensifying" role of sobre todo and the codification of "enunciative vagueness" of al menos and por lo menos. The sociolinguistic analysis showed a relatively homogeneous social distribution of sobre todo, while the employment of por lo menos is prominent among the speakers of the first age group and those of the middle-high socioeconomic group and al menos, for its part, it is used mostly by speakers of the second age group and of the low socioeconomic group. However, only the variation of por lo menos was statistically significant.

Key words: scalar operators, textual plans, polyfunctionality, chilean spanish.

\section{INTRODUCCIÓN}

En este artículo nos proponemos describir el comportamiento pragmático y sociolingüístico de tres operadores argumentativos de carácter escalar en la lengua espańola: sobre todo, al menos y por lo menos, en un corpus de 120 entrevistas sociolingüísticas realizadas a hablantes santiaguinos. Por un lado, para el análisis pragmático aplicamos el concepto de polifuncionalidad o multifuncionalidad (Bazzanella 2006) de los marcadores del discurso, es decir, la capacidad de estas unidades de activar distintos valores pragmáticos en determinados contextos. Por otro, para el análisis sociolingüístico indagamos la distribución social del empleo de estos operadores, en particular, su correlación con los factores sociodemográficos de los sujetos de la muestra. De este modo, nuestra pesquisa consta de una sección cualitativa (análisis pragmático) y de otra cuantitativa (análisis sociolingüístico). Para la primera utilizamos, en especial, herramientas teóricas provenientes del análisis del discurso oral; la segunda, por otro lado, se inscribe en las directrices teóricas y metodológicas de la sociolingüística variacionista y toma en cuenta las sugerencias de Cortés (1998) y Carbonero y Santana (2010) para el análisis cuantitativo de los marcadores discursivos. Dicho encuadre analítico de perfil sociopragmático lo hemos aplicado ya en varios estudios sobre el empleo de los marcadores del discurso en el español de Chile (San Martín 2015, 2016a, 2016b, 2016c y 2017). Nuestros objetivos específicos son: a) describir el carácter polifuncional de los operadores escalares sobre todo, al menos y por lo menos a la luz de los valores que adquieren en los distintos planos textuales y b) correlacionar su empleo con las variables independientes sexo-género, edad y grupo socioeconómico de los hablantes que conforman la muestra seleccionada. En consideración a lo anterior, con el presente estudio buscamos contribuir al estudio de los operadores escalares en el español de Chile en su modalidad oral, una dimensión privilegiada en las últimas décadas por el bullente trabajo del análisis pragmático y sociolingüístico del español coloquial. 


\section{Marco}

Según Fuentes Rodríguez, la distinción entre conectores y operadores radica en que mientras los primeros relacionan dos enunciados, los segundos introducen "modificaciones en el enunciado que afectan a todo lo expresado en él” (2009: 378). Dentro de los operadores, esta autora reconoce distintos tipos dependiendo del plano del análisis en el que incidan particularmente: enunciativos, modales, argumentativos e informativos. En palabras de Fuentes Rodríguez (2003: 83):

La distinción operador/conector debe emplearse como criterio sintáctico para ver el alcance y la función de un "marcador del discurso" [...] Cada uno de estos elementos debe caracterizarse por el ámbito o ámbitos en que se mueve: modal, enunciativo, macroestructura informativa, argumentativa [...] Y pueden actuar en varios de ellos a la vez (la cursiva es nuestra).

En esta categoría de marcadores, han sido los operadores argumentativos los que han recibido mayor atención; de hecho, Fuentes Rodríguez (2003: 81) señala que, para algunos autores, son los únicos operadores que existen. En este trabajo, suscribimos la idea de que, si bien son los planos argumentativos e informativos en donde tales unidades activan sus funciones prototípicas, existen valores potenciales que se explican, más adecuadamente, si analizamos su funcionamiento en otros planos, habida cuenta de que "las unidades discursivas funcionan (o pueden funcionar) en todos ellos” (Fuentes Rodríguez 83). En este sentido, creemos que el punto de vista de la polifuncionalidad permite develar aquellos valores potenciales contenidos en estas unidades. De acuerdo con Borreguero y López Serena, entendemos este rasgo definitorio de los marcadores como "la capacidad de asumir más de una función de naturaleza pragmática en el discurso en que aparecen” (2010: 444). A este respecto, es pertinente la distinción hecha por Bazzanella (2006: 138) entre la polifuncionalidad paradigmática (in absentia), esto es, las distintas funciones que puede desempeñar un mismo marcador en diferentes enunciados de acuerdo con su posición, entonación y contexto, y la polifuncionalidad sintagmática (in praesentia), es decir, las diferentes funciones que un marcador realiza de manera simultánea en un mismo enunciado (Borreguero y López Serena 2010: 445).

Toda vez que el criterio de posición parece no ser relevante en el comportamiento de los operadores aquí estudiados (Fuentes Rodríguez 2005), atenderemos específicamente a su polifuncionalidad sintagmática, puesto que nos permitirá dar cuenta de las funciones que simultáneamente van realizando a través de los distintos planos textuales. Desde la perspectiva pragmalingüística aquí adoptada, se entiende que tales planos surgen de una concepción contexto-dependiente de la comunicación, según la cual no solo el contexto sino también los participantes se encuentran codificados; estos, por su parte, se conceptualizan como agentes comunicativos situados. Específicamente, según Fuentes Rodríguez (2013: 26-27): 
La inscripción de la subjetividad del hablante (modalidad) o la referencia al propio acto comunicativo (enunciación) surgen de la actividad del emisor. La estructuración de la información, marcando su relevancia o focalizando segmentos, o la argumentación, para convencer, surgen de la referencia al receptor.

Nos hacemos eco de esta división que supone que hablante y oyente se inscriben en diferentes niveles: enunciativo-modal y argumentativo-informativo, respectivamente, no olvidando que es en la intersección de estos planos en donde corresponde analizar el funcionamiento pragmático de las partículas discursivas. Por último, cabe señalar que es la misma polifuncionalidad la que genera esta multidimensionalidad e interconexión de planos (Fuentes Rodríguez 2013: 27), habida cuenta de que "unidades y planos actúan de forma modular e interactiva" (2013: 18).

Por otro lado, dentro del plano argumentativo, los operadores afectan a la orientación o la fuerza argumentativa de un enunciado o de segmentos menores de enunciado (Fuentes Rodríguez 2003). Su empleo supone que el hablante busca convencer a su interlocutor de algo y, para ello, articula la información en una escala de fuerza. Siguiendo a Ducrot (1980), Fuentes Rodríguez (2016: 110) define las escalas argumentativas como "aquellas en las que los elementos o los enunciados se ordenan según su fuerza como argumentos para sostener una conclusión"2. En esta misma línea, Leonetti (1993: 111) define las escalas como un "conjunto de expresiones lingüísticas que pueden ordenarse linealmente de acuerdo con su grado de fuerza semántica o su potencial informativo". Luego, si consideramos el nivel de fuerza (+/-) que el operador escalar añade, es posible distinguir, entre otros, operadores de preferencia como sobre todo y de suficiencia como al menos y por lo menos.

Por consiguiente, como clave analítica de entrada a nuestros datos, adoptamos la matriz propuesta por Fuentes Rodríguez (2013), quien, desde una perspectiva pragmalingüística, define la comunicación textual como un proceso de construcción complejo integrado por distintos planos que "surgen de la inscripción en el contexto comunicativo" (30). Más que entidades discretas o segmentables, los planos funcionan de manera cruzada e interactiva en la constitución heterogénea de todo texto/discurso. Así, proponemos que las funciones desempeñadas por los operadores aquí estudiados pueden ser mejor descritas si atendemos a los valores específicos que comunican en relación con el propio acto comunicativo (plano enunciativo), con la inscripción de la subjetividad del hablante (plano modal), con la fuerza argumentativa (plano argumentativo) o con la focalización de determinadas unidades (plano informativo).

En síntesis, si bien la bibliografía consultada ha descrito principalmente el funcionamiento de estas partículas en tanto operadores argumentativos-informativos, y ha relegado a un lugar secundario el examen de las funciones que pueden comunicar en

\footnotetext{
${ }^{2}$ Fuentes Rodríguez $(2003,2005,2009,2013$ y 2016), la principal autora en lengua española que ha estudiado el comportamiento de los operadores argumentativos, toma, para su descripción, nociones provenientes de la Teoría de la argumentación en la lengua, tales como fuerza y orientación argumentativa (Anscombre y Ducrot 1994; Ducrot 1980 y 1986).
} 
los planos enunciativo y modal, en trabajos como el de Fuentes Rodríguez (2005) se da cuenta de su carácter complejo. En su estudio de los "operadores de preferencia", categoría en la que inscribe a sobre todo, la autora señala que la "preferencia", junto con ser una actividad enunciativa, "provoca una enfatización o relevancia informativa y argumentativa del elemento que destaca" (111). Es decir, su valor pragmático solo puede recuperarse a la luz de la intersección de los distintos planos en que este funciona. Una pregunta que guía nuestro análisis, entonces, dice relación con verificar las funciones realizadas desde la perspectiva del emisor, en términos de su subjetividad (modalidad) y de cómo refiere a su propio mensaje (enunciación), más allá de las funciones argumentativo-informativas habitualmente consideradas. Por su parte, al menos y por lo menos no han recibido un tratamiento particularizado, aunque Fuentes Rodríguez (1987) destaca el valor escalar de al menos en su trabajo sobre las partículas hasta, incluso y ni siquiera, y ambos operadores son recogidos en las tres fuentes lexicográficas consultadas (Santos Río 2003; DPDE 2008 y Fuentes Rodríguez 2009). Como se dará cuenta en nuestro análisis, estos operadores parecen comportarse como "marcadores de límite escalar" (Fuentes Rodríguez 2016), es decir, unidades que establecen una aserción aproximada o "vaga", pero entendida como suficiente desde el punto de vista del oyente. En dicho comportamiento concurren, además de las funciones escalares y focalizadoras prototípicas, valores modales de compromiso epistémico ${ }^{3}$, en particular, de mitigación del enunciado en el que operan.

\section{Método}

\subsection{Corpus}

El corpus que servirá de base para nuestro estudio corresponde a 120 entrevistas sociolingüísticas pertenecientes a la muestra de Santiago del corpus del grupo de Estudio Sociolingüístico del Español de Chile $(\mathrm{ESECH})^{4}$. El número de entrevistas antes indicado

\footnotetext{
${ }^{3}$ De acuerdo con González et al. (2017), entendemos la epistemicidad como la dimensión semántica relacionada con la evaluación, el juicio y el grado de compromiso o certeza hacia lo dicho.

${ }^{4}$ El ESECH es un grupo de investigación del Departamento de Lingüística de la Facultad de Filosofía y Humanidades de la Universidad de Chile coordinado por nosotros. Las entrevistas fueron realizadas a hablantes santiaguinos, entre 2005 y 2011, por los estudiantes en la asignatura de Sociolingüística de los programas de Licenciatura en Lengua y Literatura Hispánicas y Licenciatura en Lengua y Literatura Inglesas de la Universidad de Chile. En la situación de entrevista, los entrevistadores debían tratar de superar la "paradoja del observador" consiguiendo, de esta forma, una muestra significativa de discurso natural grabado (estilo vernáculo) de hablantes representativos de la comunidad de habla en estudio (Labov 1983). Por lo tanto, se procuró generar un clima de confianza con el objetivo de obtener el estilo de habla más espontáneo del sujeto. Así, se promovió la posibilidad de realizar la entrevista a personas cercanas al entrevistador, por ejemplo, familiares o amigos, resguardando, en todos los casos, la congruencia de estatus de los sujetos. En consecuencia, cabe destacar que las características sociodemográficas de los entrevistadores y los informantes coinciden en la gran mayoría de los casos. También se procuró que la entrevista se realizara en un lugar tranquilo, idealmente, en la casa del informante o en un lugar
} 
totaliza, aproximadamente, 116 horas de grabación, cuya transcripción fue exhaustivamente revisada, sin exclusión de ninguna de las secciones del instrumento.

\subsection{Población y muestra}

En nuestra investigación se consideró la población constituida por hombres y mujeres de la Región Metropolitana de más de 20 años $^{5}$. El cuestionario se aplicó a una muestra del tipo "muestra por cuotas con afijación uniforme" (López Morales 1994: 58). La muestra, así conformada, comprende un total de 120 entrevistas realizadas a igual número de sujetos ${ }^{6}$.

\subsection{Procedimiento de estratificación social utilizado en ESECH}

Para la estratificación de los sujetos que conforman la muestra del estudio, se empleó el sistema de adscripción de estatus social que se basa en la asignación del puntaje -que se indica entre paréntesis- a los informantes, de acuerdo con las siguientes variables: a) nivel educacional (3), b) profesión u ocupación (2) y c) comuna de residencia (1) ${ }^{7}$. A partir de la asignación de los mencionados puntajes, se distinguieron cuatro grupos socioeconómicos, definidos según los rangos siguientes: a) Medio alto (MA): 42 - 36, b) Medio (M): 35 - 27, c) Medio bajo (MB): $26-18$ y d) Bajo (B): $17-6$. El intervalo de cada rango responde a la mayor coincidencia con los puntajes que han establecido los estudios sociológicos y de mercado para la clasificación de estratos socioeconómicos, aunque con las debidas

que no le fuera extraño, a fin de garantizar su comodidad. Además, se optó por lugares sin ruido ambiental. Para mayor detalle, ver San Martín y Guerrero (2015).

${ }^{5}$ Por lo que refiere a los criterios de asignación de estatus de hablante nativo de Santiago de Chile, se aplicaron las siguientes restricciones en la selección de los sujetos: 1) haber nacido y residido en forma ininterrumpida en Santiago, 2) haber nacido y residido en Santiago la mayor parte de sus vidas, salvo por periodos que sumados no superen los cinco años y 3) haber residido en forma ininterrumpida en Santiago desde los cinco años.

${ }^{6}$ En cuanto al tamańo de la muestra, hemos optado por conformarla con un número de informantes considerado, en general, como suficiente en la bibliografía sociolingüística (cf. Trudgill y Hernández Campoy 2007, s. v. representatividad y López Morales 1994: 52). Por otro lado, cabe precisar que los grupos etarios que hemos considerado apelan a tres momentos vitales de los individuos (Blas Arroyo 2005) relacionados con el ejercicio de una profesión o actividad específicas: 1) ingreso al mercado laboral (20 a 34 años), 2) desarrollo laboral pleno ( 35 a 54 años) y 3) cesación de funciones o jubilación ( 55 años y más). En este sentido, se trabaja con etapas por las que pasa el hablante, que suelen estar vinculadas con la cultura de cada comunidad lingüística.

${ }^{7}$ La explicación en detalle de dichas variables se encuentra en Prieto (1995-1996), cuya propuesta fue perfeccionada y actualizada con base en los estudios disponibles acerca de la realidad socioeconómica chilena. Cabe precisar que el modelo de nivel socioeconómico fue adaptado de estos estudios de mercado en función de los objetivos que se propone la sociolingüística, que busca definir un perfil más bien sociocultural de la comunidad lingüística en estudio. En consideración a esto, para la estratificación socioeconómica, una de las variables más determinantes fue el nivel educacional y, por esta razón, es la que asigna el mayor puntaje. Asimismo, el nivel de ingresos demuestra muy poca relevancia para los estudios antes mencionados, por ello no fue considerado en la determinación de los perfiles socioeconómicos. 
adaptaciones, puesto que en esta muestra no se incluyen ni el grupo de la extrema pobreza ni el de la extrema riqueza (San Martín y Guerrero 2015).

\subsection{Procedimiento analitico}

Nuestro análisis de los operadores escalares seleccionados consideró la determinación de sus respectivas frecuencias de ocurrencia en las entrevistas que conforman la muestra. Asimismo, de modo coherente con nuestros objetivos, se observó la incidencia de los siguientes factores sociodemográficos de los hablantes: sexo-género, edad y grupo socioeconómico. El análisis estadístico atendió a dos niveles de observación: a) en términos descriptivos, según frecuencias absolutas y porcentajes de frecuencia y b) en términos interpretativos o inferenciales, con base en la comparación entre las medias o tendencias centrales de los marcadores más frecuentes, esto es, de aquellos con más de 25 ocurrencias ${ }^{8}$.

\section{ANÁLISIS}

En esta sección se presenta el análisis pragmático y sociolingüístico de los operadores sobre todo, por un lado, y al menos y por lo menos, por otro. En primer lugar, y en línea con el concepto de polifuncionalidad aquí acotado, a saber, "la capacidad [de los marcadores] de asumir más de una función de naturaleza pragmática en el discurso en que aparecen" (Borreguero y López Serena 2010: 444), se examinan las distintas funciones realizadas mediante sobre todo $(\$ 4.1 .1$.). Específicamente, junto con su caracterización en tanto operador argumentativo-informativo ( $\$$ 4.1.1.1.), se describe esta unidad en su comportamiento como intensificador en los planos enunciativo y modal (\$ 4.1.1.2.). Luego, se revisa la polifuncionalidad de al menos y por lo menos $(\$ 4.1 .2$.); para ello, atendemos, junto con sus valores argumentativo-informativos (\$ 4.1.2.1.), a la construcción de significados de modalidad epistémica de la que tales unidades participan en determinados contextos $(\$$ 4.1.2.2.). Esta función, que se realiza en los planos enunciativo y modal, se explica en tanto estrategia de mitigación del compromiso asertivo, particularidad que permite entenderlos como hedges. Finalmente, y en un segundo momento analítico, se presenta una descripción sociolingüística del empleo de los operadores en las 120 entrevistas analizadas. En concreto, se correlacionan sus frecuencias de uso con los factores sociales sexo-género, edad y grupo socioeconómico de los hablantes (\$ 4.2).

\footnotetext{
${ }^{8}$ Por lo que se refiere a la descripción de los procedimientos estadísticos aplicados, en este trabajo seguimos las sugerencias e indicaciones de Hernández Campoy y Almeida (2005). El paquete estadístico al que hemos recurrido para la estadística inferencial es el SPSS (Statistical Package for the Social Sciences), versión 15.0 para Windows, específicamente, la prueba Análisis de varianza ANOVA. En atención a que la distribución de los datos, en algunos casos, pudiera ser anormal, se complementará dicho análisis con su análogo de tipo no paramétrico, esto es, Anova de Kruskal Wallis. En ambos casos, el grado de significación se definirá en el 5\%, según el cual $\mathrm{p}=<0,05$ será estadísticamente significativo.
} 


\subsection{Análisis pragmático}

\subsubsection{Sobre todo como operador de preferencia}

Sobre todo es "el operador de preferencia prototípico" (Fuentes Rodríguez 2005: 109), formado a partir de la gramaticalización de un sintagma nominal (preposición + pronombre). Actúa típicamente en los planos argumentativo e informativo y, específicamente, "presupone un paradigma de elementos que cumplirían la predicación. Introduce el preferido por el hablante, situado en la posición más elevada de la escala" (Fuentes Rodríguez 2009: 322). El DPDE (2008), por su parte, lo define como aquella partícula que "destaca un elemento del discurso que se ha de tener más en cuenta que otro (expreso o sobreentendido) por constituir la parte mayor o más importante de la totalidad" (s.v. sobre todo):

1) I: teníamos más respeto al adulto mayor / sobre todo a los papás $\left(\mathrm{M} 092^{9}\right)$.

En 1) vemos que el sintagma al que acompaña sobre todo, "a los papás”, es el de mayor fuerza argumentativa (o, lo que es lo mismo, el preferido por el hablante y el ubicado en la parte más alta de la escala que presupone). Asimismo, este caso ilustra cómo sobre todo desempeña, en los planos argumentativo e informativo, una función de focalización o puesta en relieve, la que, como se verá más adelante, constituye un valor transversal a este operador. Junto con ella, la función de reinterpretación, que supone acotar una información general (en una relación semántica de hiperonimia-hiponimia), corresponde a otro "contexto específico de uso" (Fuentes Rodríguez 2005), en que sobre todo actúa como un "sobrerrealizante. Reduce la referencia pero aumenta la fuerza argumentativa. Lo dicho en el enunciado general se afirma para todas las situaciones. A continuación, la restricción introducida por sobre todo lo limita a un aspecto, elegido sin anular los demás" (2005: 110). Este valor se ilustra en 2):

2) I: sí / o sea / como todos saben Núñoa es una comuna que / sobre todo en el eje Irarrázaval ha cambiado bastante en cuanto a su paisaje (H097).

El hablante señala qué sector específicamente de la comuna "ha cambiado bastante en cuanto a su paisaje" y emplea el operador de preferencia para introducir el enunciado de mayor fuerza argumentativa, "en el eje Irarrázaval", en este caso, transmitiendo un

\footnotetext{
${ }^{9}$ El código de los sujetos tiene las siguientes equivalencias: Sexo-género= $\mathrm{H}$ (hombre) y M (mujer) y un número asignado correlativamente a las entrevistas del corpus. Para la transcripción de los ejemplos: 1) se emplearon las siguientes convenciones para señalar a los participantes de las entrevistas: $\mathrm{E}=$ entrevistador e $\mathrm{I}$ = informante; 2) cada ejemplo se transcribe en ortografía convencional, incluidos los acentos gráficos; 3) para la mejor comprensión de los ejemplos se ha suprimido el sistema de etiquetas pertenecientes al Standard Generalized Markup Language (SGML) contenidas en el Text Encoding Initiative (TEI), que se emplean en la transcripción de las entrevistas pertenecientes al ESECH, excepto las pausas.
} 
valor circunstancial-espacial, el que típicamente es recuperado en estos contextos de uso, según Fuentes Rodríguez (2005). Como mencionamos más arriba, el miembro discursivo deslindado por el operador no anula la predicación general, sino que particulariza la denotación (Ñuñoa $\rightarrow$ eje Irarrázaval), a la vez que aumenta la informatividad. Este valor también se aprecia en el ejemplo 3):

3) I: pero creo que para vacaciones es más bonito el sur / sobre todo la zona de los lagos y todas esas cosas (H162).

En este caso, la estructura de reinterpretación supone que sobre todo funciona como un sobrerrealizante que potencia la fuerza informativa de "la zona de los lagos y todas esas cosas": es esa la porción de discurso, enfatizada, la que debe considerarse para la interpretación correcta del enunciado. Asimismo, es posible apreciar cómo una misma partícula discursiva puede actualizar distintos valores: focalización (plano informativo), preferencia (plano enunciativo) y posición escalar elevada (plano argumentativo), es decir, "hay diversas macroestructuras y las unidades discursivas funcionan (o pueden funcionar) en todas ellas" (Fuentes Rodríguez 2003: 81). Cabe preguntarse, ahora, por el comportamiento de sobre todo en el plano modal (la dimensión relacionada con la subjetividad del hablante).

Siguiendo a Fuentes Rodríguez (2009: 323), el "valor enunciativo de elección por parte del hablante", que la autora sitúa en los planos modal y enunciativo (indistintamente), conlleva, además de una preferencia por el segmento de discurso al que modifica, la inscripción de la propia subjetividad del hablante (modalidad), es decir, posee carácter evaluativo. De este modo, a partir de nuestro análisis proponemos que en el empleo situado de sobre todo concurren dos características definitorias de la intensificación, esto es, escalaridad y evaluación, en línea con lo planteado por Albelda (2014). Para esta autora, la intensificación debe pensarse como una categoría pragmática realizada por determinadas formas lingüísticas, cuyo valor solo puede ser recuperado contextualmente, toda vez que corresponde a la estrategia de un hablante situado que inscribe su subjetividad en un acto particular de enunciación: "la intensificación, por tanto, no es ni está en la forma, es la inferencia que se obtiene del modo de expresión elegido" (Albelda 87; la cursiva es nuestra).

Según el rasgo de escalaridad, todo mecanismo intensificador conlleva la aserción de una escala, en tanto que sitúa en un punto extremo a un determinado elemento y, a la vez, presupone la existencia de otras posibilidades más débiles o menos informativas (2014: 83), como en el caso de sobre todo. La evaluación, por su parte, pone de relieve la idea de que "quien intensifica no solo expone un estado de cosas, sino que juzga la realidad, y con la elección de las formas lingüísticas pretende revelar ese juicio" (2014: 85), es decir, dar cuenta de su sistema de valores o punto de vista ${ }^{10}$. Así, Albelda (2014: 86) afirma que:

\footnotetext{
${ }^{10}$ En palabras de Gaviño (2014: 15), "a partir de esta relación natural entre enunciador y enunciado se vislumbra la manera en que el enunciador manifiesta su yo con respecto a lo dicho, especialmente en un registro como el coloquial, en el que la manifestación de la subjetividad y la frecuente temática en torno al yo y al tú, facilita la expresión de la modalidad".
} 
En la intensificación el hablante se hace presente en el enunciado escalar al manifestar la preferencia y elección por un punto concreto de la escala: el más alto que el hablante considera informativo tomar para comunicar que se supera lo normal o esperable en ese contexto.

En efecto, la función de sobre todo como intensificador pragmático responde a la estrategia comunicativa, siempre contextual, que este activa, toda vez que "en la intensificación se pone en juego cómo un hablante consigue dar fuerza y destacar sus intenciones para que sus destinatarios reconozcan el efecto buscado" (Albelda 2014: 81). Visto así, es evidente el cruce con los planos argumentativos e informativos: tanto la fuerza argumentativa como la enfatización provienen de la misma intención que es, en última instancia, siempre inscripción de un yo que se posiciona frente a lo que dice.

\subsubsection{Sobre todo en los planos argumentativo e informativo: escalaridad y focalización}

Como ha quedado expuesto más arriba, el comportamiento de sobre todo en los planos argumentativos e informativos ha sido del que con mayor profusión ha dado cuenta la bibliografía especializada. En efecto, es posible señalar que las funciones escalar y focalizadora de este operador constituyen los valores que típicamente comunica o, lo que es lo mismo, su "primer plano"11 de funcionamiento pragmático:

4) I: no me llama la atención la religión / ni la mormona ni la evangélica ni la católica sobre todo la evangélica me apesta / no no me llama la atención así (H002).

5) I: la verdad es que no lo pasé muy bien en la / en / en la escuela / en la básica sobre todo / no / nunca le tuve mucho carińo al tema de la básica (M073).

6) I: la debilidad mía sí / el cola de mono ahí mi marido siempre no me no me falta a mí ahí mi botellita / sobre todo para mí porque a él le gusta él toma / pero más para mí (M093).

En todos estos casos, el hablante focaliza un elemento ("la evangélica", "en la básica" y "para mí", respectivamente), a la vez que lo ubica en el punto máximo de una escala de elementos menos informativos que le siguen. Lo anterior constituye un rasgo definitorio de la escalaridad, a saber, la relatividad: esta "pone de manifiesto que existen otras alternativas inferiores que no se han elegido porque no son suficientemente informativas para los propósitos del hablante" (Albelda 2014: 84). Mientras en 4) y en 6) esas "otras alternativas inferiores" están explícitas, en 5) las opciones menos informativas deben inferirse del resto de los ciclos educativos ("enseñanza media", por ejemplo).

${ }^{11}$ De acuerdo con Fuentes Rodríguez (2013: 27), la "coexistencia de valores se produce en una jerarquía de relevancia o visibilidad, entre lo podríamos llamar primer plano y fondo”. 


\subsubsection{Sobre todo en los planos enunciativo y modal: preferencia y evaluación}

En el comportamiento argumentativo-informativo de sobre todo ya se prefigura la función que desempeña en los planos enunciativos y modales. En efecto, la puesta en relieve de un determinado elemento evidencia la elección y la "preferencia" del hablante, es decir, corresponde a un acto a través del cual este valora más un segmento discursivo que otros. La función evaluativa de sobre todo, presente en los ejemplos 7) a 10), se da en contexto de reinterpretación (general-particular):

7) I: o si tengo que participar en algún concurso con ellos / igual sí // siempre estoy / de hecho soy bien / bien humanitario / me gusta ayudar a la gente / sobre todo a los niños / me encantan los niños / (H053).

8) I: porque les gusta la televisión / les gusta la farándula y mientras más farándula tenga la televisión más gente ve las / este tipo de programas y sobre todo las mujeres // a las mujeres les fascina el pelambre // lo que se dicen unas a otras (H129).

9) I: hay que ver que hay que tener cuidado en los medios de comunicación // porque / sobre todo con los canales abiertos / porque son medios masivos (M073).

10) I: pero los oftalmólogos son una especialidad muy en falencia que se llama / o sea faltan muchos oftalmólogos sobre todo en el sistema público de salud (M186).

En consideración a lo anteriormente planteado, la función intensificadora de sobre todo deriva de su participación multidimensional, en tanto la escalaridad y la evaluación, dos rasgos que definen tal función, van de la mano de los valores de realce informativo y de preferencia enunciativa. En los ejemplos anteriores, en particular, la intensificación se articula en secuencias textuales argumentativas (constituyen "opiniones"), de modo tal que permite la inscripción de la subjetividad mediante juicios valorativos que tienen, como finalidad, convencer.

\subsubsection{Al menos y por lo menos como operadores de suficiencia}

El DPDE (2008) define almenos y por lo menos, en la función que aquí describimos ${ }^{12}$, como aquella partícula que "destaca el miembro discursivo al que afecta como información segura e implica a la vez que puede comprender otra información posible (expresa o sobreentendida), de la que no se tiene total certeza" (s.v. al menos y por lo menos), mientras

\footnotetext{
${ }^{12}$ El valor "consolatorio" de estas partículas también es recogido por el DPDE (2008). Sin embargo, dada su escasa aparición en la muestra analizada, no se considera su revisión en nuestra pesquisa. Una aproximación que profundiza en el comportamiento escalar de la partícula al menos se encuentra en Portolés (2007). Asimismo, Santos Río (2003) considera que por lo menos y al menos comparten los mismos valores escalar y argumentativo; específicamente, señala que este último, "1.-Establece un tope por abajo (eso y más). Suele actuar como subjunto [...] 2.-Establece un mínimo satisfactorio. Conlleva una sutil referencia a una porción textual previa y suele tener carácter argumentativo o asimilado" (s. v. al menos).
} 
que Fuentes Rodríguez (2009: 43) apunta que al menos "acompaña a un segmento al que sitúa en la parte baja de la escala. Este se valora positivamente y se considera suficiente por parte del hablante”. A esta definición remite en la entrada de por lo menos (2009: 272). En este estudio, consideramos que las características semántico-pragmáticas de ambos operadores los acercan a lo que Fuentes Rodríguez (2016) denomina "marcadores de límite escalar" (MLE, en adelante): unidades del tipo como máximo o como minimo que el hablante emplea "para cuantificar o asertar de forma imprecisa, marcando los límites en que se sitúa lo dicho" (2016: 106; la cursiva es nuestra).

Para los fines de nuestra pesquisa es de especial relevancia la descripción que Fuentes Rodríguez propone para los MLE como poco y como mínimo, pues dan cuenta de un funcionamiento similar al exhibido por al menos y por lo menos:

11) I: vamos a la playa por el día / pero hay que salir a tal hora / te demoras por lo menos tres horas en ir / hay que salir súper temprano (M026).

En 11) el hablante establece una escala cuyo límite inferior es "tres horas", a la vez que presupone una cantidad mayor (dirección ascendente de la escala) que debe ser inferida por el oyente: probablemente "te demoras más ${ }^{13}$ de tres horas". Siguiendo a Fuentes Rodríguez (2016: 113), "el receptor tiene que inferir cuál es la información que quiere transmitirnos el hablante, aunque de forma vaga. Este no es, pues, el responsable de lo dicho ni de la fuerza de esa aserción, sino el oyente". Como se desprende de la cita anterior, el empleo de los MLE implica que el hablante es impreciso en la entrega de la información y solo fija un límite desde el cual el oyente debe hacerse cargo de lo enunciado a través de su correcta interpretación. En otras palabras, por lo menos codifica un valor de vaguedad enunciativa o fuzziness (Fuentes Rodríguez 2016). Lo anterior podría ser indicativo de una estrategia de mitigación del compromiso asertivo, puesto que tales operadores "son términos que con frecuencia transmiten ambigüedad, imprecisión o duda, muchas veces fruto de no querer el hablante comprometerse con la exactitud de lo dicho" (2016: 111), como queda de manifiesto en 12):

12) $\mathrm{E}: \mathrm{mmm} /$ ¿es seguro este barrio?

I: este al menos sí / yo no sé / yo no he sabido que hayan aquí robos y asaltos / si eso si eso / se entiende por seguridad (H165).

En este caso, el informante presupone que puede que otros barrios no sean seguros, información con la que no se compromete, puesto que no cuenta con evidencias para señalarlo. Así, delega nuevamente en el oyente la responsabilidad de salvar la vaguedad enunciativa mediante un proceso inferencial. Sobre este análisis, y en línea con lo planteado

${ }^{13}$ Como señala Fuentes Rodríguez (2016: 117): el marcador "indica la dirección en que se da la afirmación: hacia abajo o hacia arriba. Esta orientación es contraria a la que indica la base léxica del término utilizado". 
por Fuentes Rodríguez (2016: 113), al menos y por lo menos "pueden funcionar en los otros planos: la subjetividad (hedges) o la fuente de información (shields), como intentaremos poner en evidencia en $\$$ 4.1.2.2.

\subsubsection{Al menos y por lo menos en los planos argumentativo e informativo: límite escalar}

Tal como señalamos anteriormente, al menos y por lo menos presentan un comportamiento similar al que caracteriza a los MLE estudiados por Fuentes Rodríguez (2016), en términos de que afirman un elemento y, conjuntamente, hacen inferir un contenido más informativo orientado en una dirección opuesta a la indicada por el operador. Esta cuantificación aproximada se ilustra en los ejemplos 13) al 15):

13) I: hay por lo menos una vez al año en que me doblo el mismo tobillo y todo a causa de esa vez (M154).

14) I: en en aquella época era era parte de la vida confesarse / por lo menos una vez a la semana digamos / yo yo que vengo de un colegio de curas asistía a misa todos los días / y me confesaba por lo menos una vez a la semana (H179).

15) I: he sido víctima / de al menos dos robos / en una ocasión en / allá donde vives tú / cerca de donde vives tú (M188).

En 13), por un lado, el hablante comunica, sin decirlo, que más de una vez al año se dobla el mismo tobillo, mientras que en 14) el informante implica que se confesaba más de una vez a la semana. En 15), por último, el oyente debe inferir que son más de dos robos de los cuales su interlocutor ha sido víctima.

4.1.2.2. Al menos y por lo menos en los planos enunciativo y modal: vaguedad y atenuación del compromiso asertivo

En los ejemplos que se presentan a continuación, al menos y por lo menos funcionan como partículas que, a la vez que hacen inferir una información distinta a la explicitada, contribuyen a la configuración de valores semánticos de modalidad epistémica. Así, en todos estos casos, junto con la vaguedad enunciativa que transmiten, estos operadores mitigan el grado de certeza del hablante (un valor que también podría leerse en términos de cortesía). En 16), por ejemplo, al menos es empleado por la hablante para establecer una escala cuyo límite es ella misma (“yo al menos”). Este procedimiento le permite referir una experiencia que, dado su grado de conocimiento, no es posible extender al resto de las personas, si bien es la inferencia que se activa: "yo al menos no tengo ningún / ninguna amiga de mi niñez po", esto es, "los demás, no sé":

16) I: son muchas las personas que pasan por la vida de uno / pero yo al menos no tengo ningún / ninguna amiga de mi niñez po (M045). 
Esa misma función es que la se ilustra en los ejemplos 17) al 20), en donde, de manera consistente, es el propio hablante quien se fija como límite de la escala: "a mí por lo menos" (17), "por lo menos yo creo eso" (18), "por lo menos en mi / mi percepción” (19) y "al menos en mi caso" (20):

17) I: buscan como las palabras justas y / en general escriben y pronuncian bastante bien / me ha tocado ver a mí por lo menos (H101).

18) I: qué temas se conversan / qué confianza tú les das a ellos

E: sí

I: por lo menos yo creo eso (M121).

19) sí hay cambios de un año al otro año / pero si uno saca la cuenta / las cosas son / más o menos similares / por lo menos en mi / en mi percepción / habría que tomar también en cuenta eso (H146).

20) si el problema está que / toda la gente reclama de que lo / que los tacos / que / que no pasan seguido cosas por el estilo / pero para eso la solución está en salir más temprano / al menos en mi caso (H083).

\subsection{Análisis sociolingüistico}

En esta sección presentamos los resultados de nuestro análisis cuantitativo de las frecuencias de empleo de los operadores escalares seleccionados, a fin de realizar una caracterización sociolingüística de su comportamiento. Puesto que se trata de unidades que pueden suscribirse a una categoría general, pero con claras diferencias en cuanto a su funcionamiento pragmático, más allá de encontrar regularidades en su uso, nos interesa solo mostrar ciertas tendencias. Luego de presentar los datos comparativos de su ocurrencia en el corpus de 120 entrevistas sociolingüísticas analizado, se correlacionan sus frecuencias de uso con los factores sociales sexo-género, edad y grupo socioeconómico de los hablantes de dicha muestra. Como ya se señaló más arriba, se entregarán datos en términos descriptivos (frecuencias absolutas y porcentuales) e inferenciales (contraste de medias paramétrico y no paramétrico).

La Tabla 1 muestra las frecuencias de empleo (absolutas y porcentuales) de los operadores escalares sobre todo, por lo menos y al menos en el corpus. Según estos resultados, el operador de suficiencia por lo menos es el más empleado, seguido del operador de preferencia sobre todo y por el también de suficiencia al menos. Debido a la similitud funcional y formal entre por lo menos y al menos, dicho contraste es el más pertinente para nuestro análisis. De esta manera, podemos destacar que, en nuestros materiales, el operador de suficiencia más recurrido por los hablantes fue por lo menos, a notable distancia de al menos. Con todo, creemos que también es reconocible la distancia en el empleo de ambas subfunciones: suficiencia (de por lo menos y al menos) y preferencia (de sobre todo) con un predominio de 
Tabla 1. Distribución de frecuencias absolutas y porcentajes de ocurrencia de los operadores escalares analizados en la muestra.

\begin{tabular}{|l|c|c|c|c|c|c|}
\hline \multirow{2}{*}{} & \multicolumn{2}{|c|}{ sobre todo } & \multicolumn{2}{c|}{ por lo menos } & \multicolumn{2}{c|}{ al menos } \\
\cline { 2 - 7 } & $\mathrm{N}$ & $\%$ & $\mathrm{~N}$ & $\%$ & $\mathrm{~N}$ & $\%$ \\
\hline Hombres & 47 & 55,95 & 94 & 59,87 & 26 & 57,78 \\
\hline Mujeres & 37 & 44,05 & 63 & 40,13 & 19 & 42,22 \\
\hline $20-34$ & 27 & 32,14 & 70 & 44,59 & 9 & 20 \\
\hline $35-54$ & 29 & 34,52 & 41 & 26,11 & 22 & 48,89 \\
\hline 55 y más & 28 & 33,34 & 46 & 29,30 & 14 & 31,11 \\
\hline Bajo & 24 & 28,57 & 22 & 14,01 & 23 & 51,11 \\
\hline Medio bajo & 18 & 21,43 & 23 & 14,65 & 11 & 24,44 \\
\hline Medio & 14 & 16,67 & 33 & 21,02 & 2 & 4,44 \\
\hline Medio alto & 28 & 33,33 & 79 & 50,32 & 9 & 20 \\
\hline TOTAL & $\mathbf{8 4}$ & $\mathbf{1 0 0}$ & $\mathbf{1 5 7}$ & $\mathbf{1 0 0}$ & $\mathbf{4 5}$ & $\mathbf{1 0 0}$ \\
\hline
\end{tabular}

la primera por sobre la segunda, si bien es cierto que está representada por dos operadores y no por solo uno.

Por otro lado, por lo que refiere a la correlación con las características sociodemográficas de los sujetos, en términos descriptivos, podemos desprender de la Tabla 1 que se da una distribución social relativamente homogénea en el uso del operador de preferencia sobre todo, sin diferencias notables según el sexo-género, el grupo etario ni el grupo socioeconómico de los sujetos de la muestra. No obstante, una eventual excepción a esta tendencia sea el descenso en su empleo por parte del grupo socioeconómico medio en comparación con el medio alto. Por el contrario, el operador de suficiencia por lo menos presenta una distribución de uso desigual en el corpus, de acuerdo con el cual se da más en hombres que en mujeres; asimismo, muestra un empleo predominante entre los informantes del grupo etario más joven; igualmente, es preferido por los sujetos del grupo socioeconómico medio alto. Por su parte, el operador de suficiencia al menos es utilizado de modo mayoritario por hablantes del segundo grupo etario (adultos de 35 a 54 años), así como es más frecuente en las entrevistas de los hablantes del grupo socioeconómico bajo ${ }^{14}$.

\footnotetext{
${ }^{14}$ A este respecto, como se señaló en la nota 3, las características sociodemográficas de los entrevistados y los informantes de la muestra son coincidentes; por lo tanto, los rasgos sociales de los interlocutores no operan como una variable determinante en el análisis.
} 
En relación con el análisis estadístico inferencial (paramétrico y no paramétrico) del empleo de los operadores seleccionados, este arrojó solo un resultado significativo, específicamente, la asociación entre el empleo del operador de suficiencia por lo menos y el grupo socioeconómico de los sujetos. Dicho resultado se muestra en el gráfico 1 que presentamos a continuación:

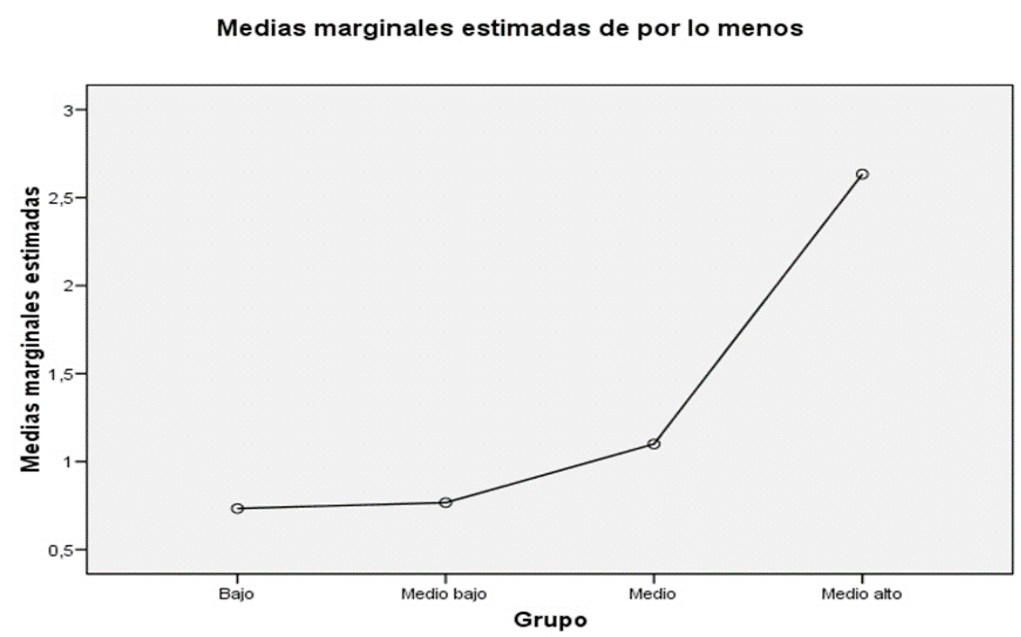

Gráfico 1. Medias marginales estimadas del operador escalar por lo menos según el grupo socioeconómico de los sujetos.

El análisis de varianza (ANOVA) de las diferencias entre medias de los grupos socioeconómicos respecto del operador de suficiencia por lo menos arroja los siguientes datos: bajo $(0,733)$, medio bajo $(0,767)$, medio $(1,100)$ y medio alto $(2,633)$, los que son significativos estadísticamente $(\mathrm{F}=7,227 ; \mathrm{p}=0,000)$. La prueba de Kruskal-Wallis indica los siguientes rangos promedio: bajo $(54,18)$, medio bajo $(50,30)$, medio $(57,67)$ y medio alto $(79,85)$; igualmente, señala que estas diferencias son significativas, ya que Chi-cuadrado= 14,$951 ; \mathrm{p}=0,002$.

Como se desprende de estos datos, el operador de suficiencia por lo menos se emplea mucho más en el discurso de los sujetos del grupo socioeconómico más alto configurándose, incluso, un patrón de estratificación abrupto con una curva creciente que se ilustra en el gráfico. Desde el punto de vista sociolingüístico, podríamos interpretar este comportamiento por parte de los hablantes del grupo medio alto como una característica más elaborada de su discurso, motivada por la necesidad de aclarar más sus puntos de vista, así como hemos hecho anteriormente (San Martín 2017) con el predominio de los reformuladores explicativos en dicho grupo socioeconómico. La orientación hacia la expresión de opiniones 
desde una perspectiva más personal podría estar en la base del predominio de por lo menos, en tanto recurso de mitigación enunciativa, en el habla de los sujetos del grupo medio alto. Finalmente, por lo que refiere a la intersección entre factores que ofrece el análisis de la varianza de ANOVA, la única intersección que dio un resultado significativo fue el cruce entre edad y sexo-género de los sujetos con respecto al empleo de por lo menos (ANOVA: $F=4,606 ; p=0,012)$. La varianza es la siguiente: I) 20-34: Hombre (2,750), Mujer $(0,750)$; II) 35-54: Hombre (0,900), Mujer (1,150); III) 55 y más años: Hombre (1,050), Mujer $(1,250)$ y puede representarse en el gráfico 2 , a continuación:

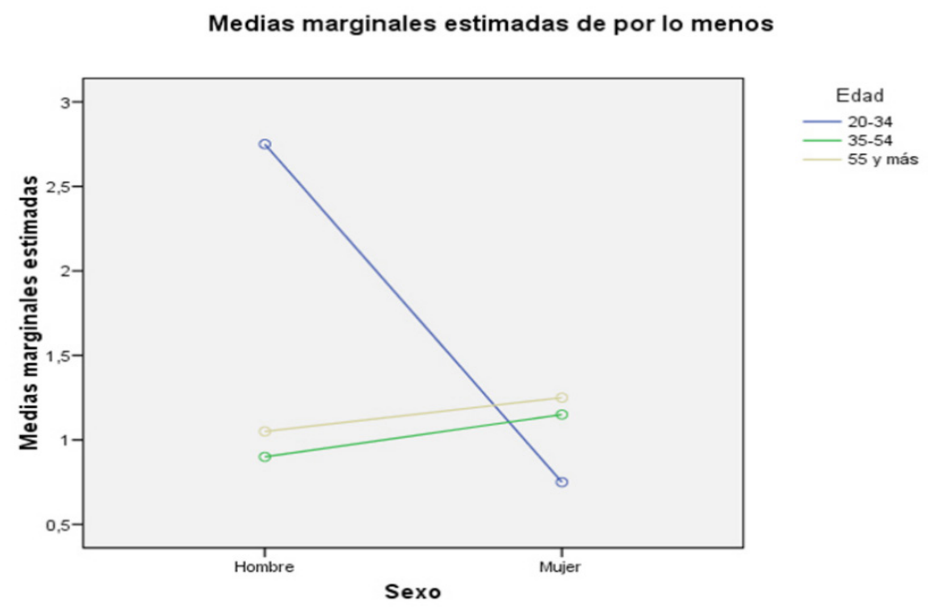

Gráfico 2. Medias marginales estimadas del operador escalar por lo menos según el sexo y la edad de los sujetos.

Los hombres del grupo de 20-34 años usan mucho más por lo menos que las mujeres del mismo grupo etario. En cambio, en el segundo y tercer grupo etario (35-54 y 55 y más años) son las mujeres quienes tienden a emplear más por lo menos que los hombres, aunque las diferencias de uso son notablemente menores. La profusión en el empleo de por lo menos como operador de suficiencia, por parte de los hombres más jóvenes de la muestra, probablemente, podría llevarnos a concluir que la construcción de su discurso es menos asertiva o más imprecisa que el de las mujeres del mismo grupo, debido a que se trata de un recurso de mitigación y vaguedad en la enunciación. Este aspecto resulta de interés, habida cuenta de los prejuicios que acerca de este tópico han existido con respecto al habla femenina, a la que en términos populares se ha caracterizado como más insegura. Según Coates (2009: 26), dicha asociación se relaciona con la primera de las perspectivas de los estudios de género, el llamado enfoque del déficit, que surgió con Lakoff (1975) para 
quien el lenguaje femenino presentaba deficiencias o debilidades respecto del masculino, entre ellas, la inseguridad o falta de asertividad. Esta asociación responde a un estereotipo que no encuentra sustento empírico, de acuerdo con Coates (2009), y que se desprende de un prejuicio acerca del habla femenina. Por otra parte, también nos parece relevante la necesidad recalcada por Cameron (2005) de relacionar la variable género con otras variables como la edad del hablante, puesto que las diferencias entre el habla de mujeres y hombres nunca es completa, debido a que es más o menos intensa en distintas etapas de la vida de las personas. Como ya señalamos, con respecto a por lo menos, en tanto operador pragmático específico, nuestros datos revelan que las diferencias en su empleo en el discurso de hombres y mujeres se da en los sujetos jóvenes, lo que confirma la utilidad de analizar también la interacción entre las variables.

\section{ConClusiones}

Nuestro análisis pragmático y sociolingüístico de los operadores escalares sobre todo, al menos y por lo menos, en el español hablado de Santiago, nos permitió dar cuenta de su complejidad y diversidad relativas. Por una parte, desde un punto de vista pragmático, confirmamos el carácter polifuncional de estos operadores que evidencian valores en los distintos planos de la actividad discursiva, a saber, argumentativo, enunciativo, informativo y modal. Asimismo, más allá de los rasgos que los aúnan, esto es, escalaridad, focalización y reinterpretación, comprobamos efectos de sentido menos trabajados en la bibliografía consultada, como son la función intensificadora de sobre todo y la codificación de vaguedad enunciativa de almenos y por lo menos. Por otra parte, desde una aproximación sociolingüística, verificamos que, en términos descriptivos: a) sobre todo se distribuye socialmente de modo relativamente homogéneo; b) por lo menos se emplea más por los hablantes del primer grupo etario y los del grupo socioeconómico medio alto y c) al menos se usa mayormente por hablantes del segundo grupo etario y del grupo socioeconómico bajo. No obstante, en términos inferenciales, solo las diferencias en el empleo de por lo menos arrojaron índices significativos, estadísticamente, los que confirman que se usa más por sujetos del grupo socioeconómico medio alto y por hombres jóvenes. Dichas diferencias las interpretamos como el reflejo de la necesidad de los hablantes del mencionado grupo de expresar sus ideas desde un punto de vista más personal, lo que redunda en el empleo de recursos de mitigación como por lo menos, así como una señal de que el estereotipo del habla femenina como menos asertiva debe relativizarse, puesto que los hombres jóvenes de nuestros materiales también recurren con frecuencia al mencionado recurso. Sin embargo, es preciso reconocer que nuestros hallazgos se limitan solo al empleo de un operador en específico, por lo que se requiere de un análisis más extensivo de procedimientos de mitigación discursiva, a fin de confirmar estos resultados. Para finalizar, quisiéramos destacar que, en términos generales, el empleo de los tres operadores aquí analizados parece ser bastante cercano a su caracterización en las fuentes de carácter panhispánico disponibles. En esta línea analítica, 
nuestros hallazgos constituyen un aporte que busca incentivar un estudio contrastivo de estos operadores argumentativos en otras variedades regionales del espańol, con el propósito de corroborar, empíricamente, su extensión y los condicionamientos de su empleo, tal como hemos hecho en este artículo.

\section{Obras Citadas}

Anscombre, Jean y Oswald Ducrot. 1994. La argumentación en la lengua. Madrid: Gredos. Albelda Marco, Marta. 2014. "Escalaridad y evaluación: rasgos caracterizadores de la intensificación pragmática”. En Elissa Putska y Stefanie Goldschmitt (eds.) Emotionen, Expressivität, Emphase. Berlín: Erich Schmidt Verlag. 79-94.

Bazzanella, Carla. 2006. "Segnali discorsivi e sviluppi conversazionali". En Federico Albano Leoni y Rosa Giordano (eds.). Italiano parlato. Analisi di un dialogo. Napoli: Liguori. 137-157.

Blas Arroyo, José Luis. 2005. Sociolingüistica del español. Desarrollos y perspectivas en el estudio de la lengua española en contexto social. Madrid: Cátedra.

Borreguero, Margarita y Araceli López Serena. 2010. "Los marcadores del discurso y la variación lengua hablada vs. lengua escrita”. En Óscar Loureda y Esperanza Acín (coords.). Los estudios sobre marcadores del discurso, hoy. Madrid: Arco/Libros. 415495.

Cameron, Deborah. 2005. "Aging and gendering”. Language in Society 34: 23-61.

Carbonero, Pedro y Juana Santana. 2010. "Marcadores del discurso, variación dialectal y variación social”. En Loureda, Óscar y Estrella Acín (coords.). Los estudios sobre marcadores del discurso, hoy. Madrid: Arco Libros. 497-521.

Coates, Jennifer. 2009. Mujeres, hombres y lenguaje. México: Fondo de Cultura Económica.

Cortés, Luis. 1998. "Marcadores del discurso y análisis cuantitativo". En María Martín Zorraquino y Estrella Durán (coords.). Los marcadores del discurso. Teoría y análisis. Madrid: Arco/Libros. 143-160.

$\mathrm{DPDE}=$ Briz, Antonio, Pons, Salvador y José Portolés (coords.). 2008. Diccionario de partículas discursivas del español. En línea: www.dpde.es.

Ducrot, Oswald. 1980. Les échelles argumentatives. Paris: Les Éditions de Minuit. . 1986. El decir y lo dicho. Barcelona: Paidós.

Fuentes Rodríguez, Catalina. 1987. "Pragmática y relación intratextual: el caso de 'hasta', 'incluso' y 'ni siquiera”. ELUA 4: 159-176. 2003. "Operador/conector, un criterio para la sintaxis discursiva". RILCE 19.1: 61-85. 2005. "Operadores de preferencia". ELUA 19: 107-136. . 2009. Diccionario de conectores y operadores del español. Madrid: Arco/Libros. . 2013. "La gramática discursiva: niveles, unidades y planos de análisis". Cuadernos AISPI 2: 15-36. 
. 2016. "Los marcadores de límite escalar: argumentación y 'vaguedad' enunciativa”. RILCE 32.1: 106-133.

Gaviño Rodríguez, Victoriano. 2014. "Las actitudes del enunciador y su codificación lingüística en partículas discursivas”. Español Actual 102: 13-35.

González, Montserrat, Paolo Roseano, Joan Borràs-Comes y Pilar Prieto. 2017. "Epistemic and evidential marking in discourse: Effects of register and debatability". Lingua 186: 68-87.

Hernández Campoy, Juan Manuel y Manuel Almeida. 2005. Metodología de la investigación sociolingüistica. Málaga: Editorial Comares.

Labov, William. 1983. Modelos sociolingüísticos. Madrid: Cátedra.

Lakoff, Robin. 1975. Language and Woman's Place. Nueva York: Harper \& Row.

Leonetti, Manuel. 1993. "Implicaturas generalizadas y relevancia”. Revista Española de Lingüistica 23.1: 107-139.

López Morales, Humberto. 1994. Métodos de investigación lingüistica. Salamanca: Ediciones Colegio de España.

Portolés Lázaro, José. 2007. “Escalas argumentativas aditivas. Pruebas del español”. Spanish in Context 4.2: 135-157.

Prieto, Luis. 1995-1996. "Análisis sociolingüístico del dequeísmo en el habla de Santiago de Chile". Boletín de Filología XXXV: 379-452.

San Martín, Abelardo. 2015. "Variantes y equivalentes funcionales de al final: los reformuladores de recapitulación en el habla santiaguina”. Revista de Lingüistica Teórica y Aplicada 53.2: 97-119.

. 2016a. "Los marcadores de reformulación en el español oral de Santiago de Chile: análisis discursivo y sociolingüístico”. Oralia 19: 283-324.

. 2016b. "Análisis sociolingüístico de los reformuladores de rectificación en el habla santiaguina”. Literatura y Lingüistica 33: 241-264.

. 2016c. "Los reformuladores de distanciamiento en el habla santiaguina: igualy sus equivalentes funcionales”. Onomázein 34: 261-277.

. 2017. "Análisis sociolingüístico de los reformuladores de explicación en el español hablado de Santiago de Chile”. Revista Signos. Estudios de Lingüistica 50.93: 124-147.

San Martín, Abelardo y Silvana Guerrero. 2015. "Estudio Sociolingüístico del Español de Chile (ESECH): Recogida y estratificación del corpus de Santiago”. Boletín de Filologia L. 1: 221-247.

Santos Río, Luis. 2003. Diccionario de partículas. Salamanca: Luso-Española de Ediciones. Trudgill, Peter y Juan Manuel Hernández Campoy. 2007. Diccionario de sociolingüistica. Madrid: Gredos. 